\title{
Deep brain stimulation of the subthalamic nucleus in Parkinson's disease: effects of variation in stimulation parameters
}

\author{
M Rizzone, M Lanotte, B Bergamasco, A Tavella, E Torre, G Faccani, A Melcarne, \\ L Lopiano
}

\begin{abstract}
Objective-To investigate the relation between the variation of the parameters of stimulation and the clinical effectiveness in parkinsonian patients treated with deep brain stimulation of the subthalamic nucleus (STN), to provide information on the electrical parameter setting and the mechanism of action of deep brain stimulation. Methods-Ten patients with Parkinson's disease bilaterally implanted in the STN were studied. For every patient the intensity of the stimulus necessary to obtain the disappearance of contralateral wrist rigidity (required clinical effect, RCE) and the side effect threshold in 20 different conditions of stimulation, coupling four pulse width values $(60,120,210,450 \mu \mathrm{s})$ with five rate values $(10,50,90,130,170$ Hz) were determined. All the patients were tested after a 12 hour withdrawal of antiparkinsonian drugs, and the clinical evaluation was double blind.

Results-In all the patients it was impossible to obtain the RCE using 10 and $50 \mathrm{~Hz}$ stimulus rates. For all the other stimulus rate values, the intensity-pulse width curves (IPWCs) for the RCE and for the side effect threshold showed a hyperbolic trend. For every pulse width value, increasing the rate from 90 to 130 and to 170 $\mathrm{Hz}$ progressively decreased the intensity of the stimulus necessary to reach the RCE, but the differences were not significant. Within the same rate value, the progressive reduction of the stimulus intensity necessary to obtain the RCE, obtained with the lengthening of the pulse width was significant $(p<0.05)$ only comparing 60 with $210 \mu$ s and 60 with $450 \mu \mathrm{s}$. Conclusions-The findings give some useful indications for the electrical parameter setting in deep brain stimulation of the STN, and some information about the mechanism of action of deep brain stimulation.
\end{abstract}

(F Neurol Neurosurg Psychiatry 2001;71:215-219)

Keywords: Parkinson's disease; subthalamic nucleus; deep brain stimulation

Deep brain stimulation of the subthalamic nucleus (STN) has been shown to be an effective therapeutic option for advanced Parkinson's disease. ${ }^{1-4}$ The hyperactivity of STN neurons plays an important part in the pathophysiology of Parkinson's disease symptoms. ${ }^{5-7}$ Through chronic high frequency electrical stimulation it is possible to achieve a functional inhibition of the STN neurons, miming the effect of a lesion, ${ }^{89}$ with a consequent relevant improvement of the cardinal symptoms of the illness (tremor, rigidity, and akinesia). ${ }^{10}$ Moreover, this allows a significant reduction of dopaminergic drugs, with an improvement in drug induced dyskinesias. $^{1-3}$

The mechanism of action of deep brain stimulation is still unknown ${ }^{10-12}$; the characteristics of the electrical stimulus, particularly the rate, ranging between 100 and $200 \mathrm{~Hz},{ }^{13}$ make a depolarising chronic effect unlikely. ${ }^{14}{ }^{15}$ The synchronous and massive stimulation of an extensive neuronal pool could result in a disrupted output, leading to a functional block of the system; alternatively, the reduction of the firing rate of the hyperactive STN neurons could be explained by a preferential activation of the inhibitory GABAergic fibres projecting to the nucleus. ${ }^{141617}$

One of the advantages of the deep brain stimulation technique is the possibility of varying the parameters of the stimulation (rate, pulse width, voltage) to obtain the best clinical improvement, avoiding side effects. ${ }^{18}$ At present, because of the lack of knowledge about the exact mechanism of action, few indications are available about the role of the different parameters of stimulation on the effectiveness of deep brain stimulation of the STN ${ }^{10}{ }^{14}$; therefore, the electrical parameter setting for implanted patients is still not standardised.

The main objective of this study was to investigate the relation between the variation of the parameters of stimulation (rate, pulse width, voltage) and the clinical effectiveness of deep brain stimulation of the STN in patients with Parkinson's disease, to identify the pattern of stimulation capable of determining the best therapeutic window (the higher difference between the threshold for the clinical effectiveness and for the onset of side effects). For this purpose it was necessary to evaluate a constant clinical effect suitable for analysing the relation between the parameters of STN stimulation. In addition, this study may provide information about the mechanism of action of deep brain stimulation.

\section{Patients and methods}

PATIENTS

Ten patients with advanced Parkinson's disease were examined after the bilateral implant of a 
Table 1 Mean (SD) clinical characteristics of the patients

\begin{tabular}{lc}
\hline Sex & 6 men; 4 women \\
Age (y) & $62.3(6.4)$ \\
Duration of disease (y) & $15.9(4.7)$ \\
Duration of levodopa treatment (y) & $14.6(5.1)$ \\
Duration of motor fluctuations (y) & $6.5(3.6)$ \\
Duration of dyskinesias (y) & $6.0(3.5)$ \\
Hoehn and Yahr stage-on & $2.5(0.4)$ \\
Hoehn and Yahr stage-off & $4.0(0.3)$ \\
Total UPDRS score-on & $37.6(11.6)$ \\
Total UPDRS score-off & $94.6(14.4)$ \\
UPDRS part II-on & $7.4(4.1)$ \\
UPDRS part II-off & $26.7(3.7)$ \\
UPDRS part III-on & $19.1(8.0)$ \\
UPDRS part III-off & $56.4(11.6)$ \\
Levodopa preoperative daily dosage (mg) & $956.2(541.0)$ \\
&
\end{tabular}

quadripolar electrode in the STN (DBS-3389, Medtronic, Minneapolis, MN, USA). The implant was placed using a system of fusion of the CT and MRI images (Stereoplan, Radionics, USA) to detect the position of the STN in relation to the mid-commisural point; in addition, intraoperative neuronal electrical activity recording and macrostimulation were performed. Patients had been operated on at least 3 months before the study to avoid the interference of a surgical microlesive effect in the evaluation of deep brain stimulation.

Characteristics of the patients are shown in table 1. The mean age of the patients, four women and six men, was 62.3 years (range 51-70 years); the duration of the disease was 15.9 years, with an average duration of levodopa therapy of 14.6 years.

The average daily dosage of levodopa in the presurgical period was $956.2 \mathrm{mg} /$ day. The average Hoehn and Yahr stage was 2.5 in the on phase and 4.0 in the off phase. In the preoperative period patients were evaluated with the unified Parkinson's disease rating scale (UPDRS): the average score in the UPDRS part III (motor evaluation) was 19.1 in the on condition and 56.4 in the off condition. All patients gave their informed consent to the study.

EXPERIMENTAL PROTOCOL

To assess the clinical effectiveness of the stimulation we tested subjectively the wrist rigidity contralateral to the stimulated side. We considered the disappearance of wrist rigidity with the patient resting as the clinical constant effect to obtain (required clinical effect, RCE).

Each patient was examined at one side only, generally the most compromised one, because of the time necessary for the execution of the study. For the test, we used the contact of the quadripolar electrode previously identified as the most effective in the control of parkinsonian symptoms, with a monopolar cathodic configuration for the stimulation (contact of the electrode $=$ cathode; pulse generator $=$ anode) .

At least 12 hours before the beginning of the study all antiparkinsonian drugs were stopped, and the patient continued to be bilaterally stimulated. Both of the pulse generators were deactivated 1 hour before the beginning of the test, to avoid a possible after effect of the stimulation; then we tested the patients in the absence of stimulation to define the baseline condition of wrist rigidity, and of upper limb akinesia and rest tremor (UPDRS part III: items $22,23-24-25 ; 20)$. During the whole study the pulse generator of the examined side remained off, to avoid the overlapping of a clinical effect related to omolateral STN stimulation, and the patients lay comfortably on a bed during the examination.

The pulse generator was activated contralateral to the examined side, on the most effective contact. To assess the effect of variation of the stimulation parameters we tested the patients in 20 different conditions, coupling four different pulse width values $(60,120,210,450 \mu \mathrm{s})$ with five rate values $(10,50,90,130,170 \mathrm{~Hz})$. For each condition the pulse width and the rate were maintained constant during clinical evaluation, and we progressively increased only the voltage of the stimulus with $0.1 \mathrm{~V}$ steps starting at $0.1 \mathrm{~V}$. During the test we changed the voltage of the stimulus because the pulse generators are voltage constant and not intensity constant; the relative values of the current intensity were then calculated on the basis of the impedance values of the contacts tested using the nominal electrical parameters $(1 \mathrm{~V}$, $210 \mu \mathrm{s}, 30 \mathrm{~Hz})$.

For every condition, the voltage was increased until the RCE was reached; the corresponding value of current intensity was calculated and plotted on a diagram, drawing an intensity-pulse width curve (IPWC) for every rate value. When the RCE was reached, the patient's rest tremor and akinesia were scored. Afterwards, the voltage was further increased until the onset of persistent side effects, and the corresponding value of current intensity was calculated.

The pulse generator was deactivated before every change of condition, until the reappearance of the contralateral baseline wrist rigidity. During the examination the order of the 20 different conditions of stimulation was random, and all the tests were performed with either the patient or the examiner unaware of the conditions of stimulation.

Statistical analysis was by Friedman's test and the Student-Newman-Keuls test for multiple comparison; statistical significance was indicated by a $\mathrm{p}$ value $\leqslant 0.05$.

\section{Results}

CLINICAL EFFICACY

In the baseline condition the wrist rigidity mean score on the examined side was $2.6 / 4$ (SD 0.6) (UPDRS III item 22).

Table 2 shows the mean values of intensity of the stimulus giving the RCE in the 20 different conditions of stimulation rate and pulse width. With a stimulus rate of 10 and $50 \mathrm{~Hz}$ it was impossible to obtain the RCE with any pulse width apart from one patient that reached the RCE, with a rate of $50 \mathrm{~Hz}$.

With a stimulus rate of 90,130 , or $170 \mathrm{~Hz}$ it was nearly always possible to obtain the RCE whatever pulse width was set; in two patients the onset of side effects made it impossible to reach the RCE with a stimulus rate of $90 \mathrm{~Hz}$ and a pulse width of 210 and $450 \mu \mathrm{s}$. 
Table 2 Mean (SD) values of intensity $(m A)$ corresponding to the reach of the required clinical effect (RCE)

\begin{tabular}{llll}
\hline \multicolumn{4}{l}{ Rate $(\mathrm{Hz})$} \\
\cline { 2 - 4 } Pulse width $(\mu \mathrm{s})$ & 90 & 130 & 170 \\
\hline 60 & $1.7(0.6)$ & $1.4(0.3)$ & $1.3(0.4)$ \\
120 & $1.3(0.6)$ & $1.1(0.3)$ & $1.0(0.3)$ \\
210 & $1.1(0.4)$ & $1.0(0.3)$ & $0.8(0.3)$ \\
450 & $0.9(0.4)$ & $0.8(0.3)$ & $0.7(0.3)$ \\
\hline
\end{tabular}

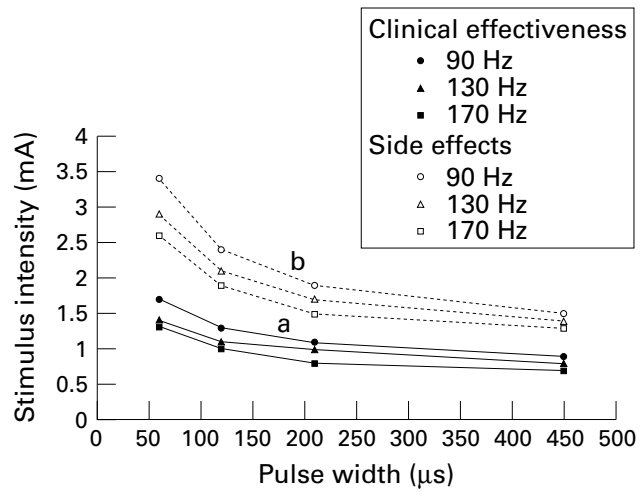

Figure 1 Intensity-pulse width curves for the different stimulus rate values $(90,130$, or $170 \mathrm{~Hz})$. (a) Stimulus intensity necessary to obtain the required clinical effect; (b) stimulus intensity relative to the side effect threshold.

The trend of the IPWCs, looking at the constancy of the clinical effect, is shown in figure 1a. For every stimulus rate $(90,130,170 \mathrm{~Hz})$ and pulse width $(60,120,210,450 \mu \mathrm{s})$ the intensity of the stimulus necessary to obtain the RCE was identified, getting an IPWC similar for each stimulus rate, with a hyperbolic trend. For every pulse width value the increase of the stimulus rate from $90 \mathrm{~Hz}$ to $130 \mathrm{~Hz}$ and to 170 $\mathrm{Hz}$ led to a better clinical effect-namely a reduction of the intensity of the stimulus necessary to obtain the RCE. None of these differences were, however, significant.

Within the same stimulus rate value, the larger the pulse width the greater the effectiveness of the stimulation. These pulse width related differences on clinical efficacy were significant comparing 60 with $210 \mu$ s and 60 with $450 \mu$ s for every stimulation rate value.

Only four patients showed rest tremor in the baseline condition on the examined side, with an average score of 2.0/4 (SD 0.7) (UPDRS III item 20). In all the experimental conditions in which it was possible to obtain the RCE on wrist rigidity we also found an improvement of the rest tremor with respect to the baseline, whereas no substantial differences were found between the different conditions (table 3). Average baseline akinesia score for the examined upper limb was 6.6/12 (SD 2.9) (UPDRS III items 23, 24, 25). Also this symptom significantly improved when the RCE for wrist rigidity was reached, and no significant differences were found between the different experimental conditions (table 4).

SIDE EFFECTS

Table 5 shows the mean values of the stimulus intensity corresponding to the side effect threshold in the different experimental conditions.
Table 3 Mean (SD) for tremor of the examined upper limb (UPDRS III, item 20) in the different experimental conditions. The scores are relative to the current intensity that determined the reaching of the RCE on wrist rigidity. Data are relative to the four patients that presented rest tremor in the baseline condition

\begin{tabular}{llll}
\hline \multirow{4}{*}{$\begin{array}{l}l \\
\text { Pulse width }(\mu s)\end{array}$} & 90 & 130 & 170 \\
\cline { 2 - 4 } & $0.4(0.5)$ & $0.4(0.5)$ & $0.3(0.3)$ \\
60 & $0.4(0.5)$ & $0.3(0.3)$ & $0.4(0.3)$ \\
210 & $0.3(0.3)$ & $0.4(0.5)$ & $0.3(0.3)$ \\
450 & $0.4(0.3)$ & $0.3(0.3)$ & $0.4(0.5)$ \\
\hline
\end{tabular}

RCE $=$ Required clinical effect.

Table 4 Mean (SD) scores for akinesia of the examined upper limb (UPDRS III items 23, 24, 25) in the different experimental conditions. The scores are relative to the current intensity that determined the reaching of the RCE on wrist rigidity

\begin{tabular}{llll}
\hline \multirow{4}{*}{ Pulse width $(\mu \mathrm{s})$} & \multicolumn{2}{l}{ Rate $(\mathrm{Hz})$} \\
\cline { 2 - 4 } & 90 & 130 & 170 \\
\hline 60 & $2.6(2.0)^{\star}$ & $2.7(2.0)^{\star}$ & $2.6(1.9)^{\star}$ \\
120 & $2.6(2.2)^{\star}$ & $2.5(2.1)^{\star}$ & $2.5(2.1)^{\star}$ \\
210 & $2.7(2.3)^{\star}$ & $2.6(2.0)^{\star}$ & $2.6(2.0)^{\star}$ \\
450 & $2.5(2.0)^{\star}$ & $2.6(2.0)^{\star}$ & $2.5(2.1)^{\star}$
\end{tabular}

${ }^{\star} \mathrm{p}<0.05 v$ baseline. $\mathrm{RCE}=$ Required clinical effect.

Table 5 Mean (SD) values of intensity $(m A)$ corresponding to the side effect threshold

\begin{tabular}{llll}
\hline \multirow{4}{*}{ Pulse width $(\mu \mathrm{s})$} & 90 & 130 & 170 \\
\cline { 2 - 4 } 60 & $3.4(0.5)$ & $2.9(0.6)$ & $2.6(0.7)$ \\
120 & $2.4(0.6)$ & $2.1(0.5)$ & $1.9(0.4)$ \\
210 & $1.9(0.4)$ & $1.7(0.4)$ & $1.5(0.3)$ \\
450 & $1.5(0.4)$ & $1.4(0.3)$ & $1.3(0.3)$ \\
\hline
\end{tabular}

The side effects found at a $10 \mathrm{~Hz}$ or $50 \mathrm{~Hz}$ stimulus rate were qualitatively different from the side effects obtained at $90 \mathrm{~Hz}, 130 \mathrm{~Hz}$, or $170 \mathrm{~Hz}$. At $10 \mathrm{~Hz}$ and $50 \mathrm{~Hz}$ the most frequent side effects, restricted to the side contralateral to the stimulated one, were the appearance of or the worsening of tremor and the onset of myoclonic jerks. With a stimulus rate of $90 \mathrm{~Hz}$, $130 \mathrm{~Hz}$, or $170 \mathrm{~Hz}$ the side effects noticed were paraesthesias, muscle contractions, and dyskinesias contralateral to the stimulated side.

Figure $1 \mathrm{~B}$ shows the IPWCs relative to the side effect threshold. The rate increase from 90 $\mathrm{Hz}$ to $170 \mathrm{~Hz}$ did not change the type of curve, but a progressive reduction of the side effect threshold value was evident. The differences between the side effect intensity threshold at the different stimulus rate never reached significance within the same pulse width value. On the contrary, the changes of the pulse width values were responsible for greater differences between the side effect threshold values; for every rate value, the increase in the pulse width from 60 to $210 \mu \mathrm{s}$, from 60 to $450 \mu \mathrm{s}$, and from 120 to $450 \mu$ s led always to a significant reduction of the side effect intensity threshold.

\section{THERAPEUTIC WINDOWS}

We defined the therapeutic window as the difference between the intensity threshold of the stimulus for onset of the side effects and the intensity value necessary to obtain the RCE in the same experimental condition. 


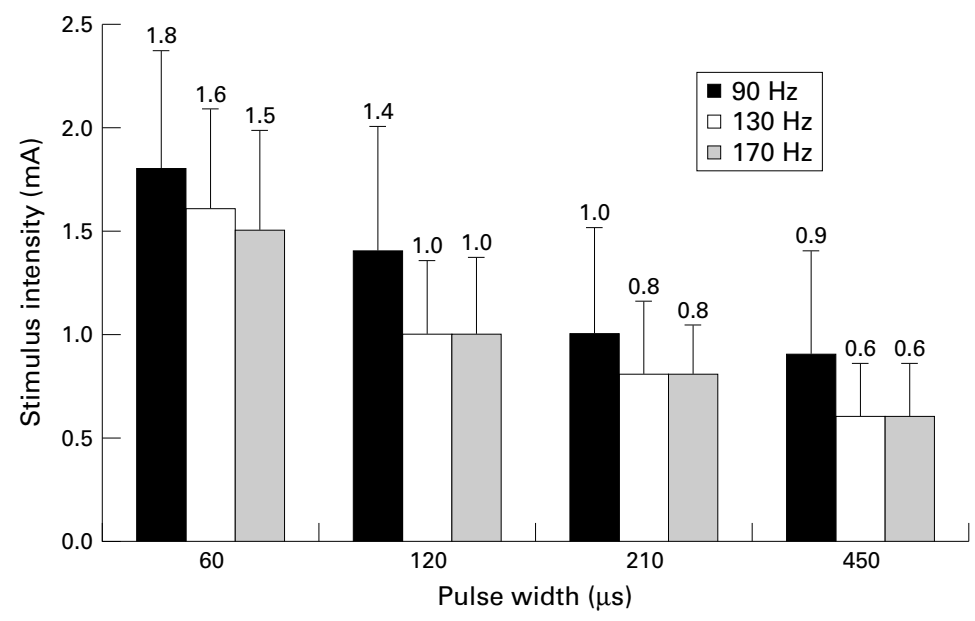

Figure 2 Therapeutic windows (difference between the intensity threshold of the stimulus for the onset of side effects and the intensity value necessary to obtain the required clinical effect (RCE) in the different experimental conditions.

Figure 1 shows, for every pulse width value, the difference between the IPWC relative to the clinical effect and the IPWC of the threshold for side effects. This difference, which represents the therapeutic window, is clinically relevant because it is the expression of the range of electrical parameters useful for clinical STN stimulation.

Figure 2 shows the therapeutic windows relative to the different pulse width and rate values. The increase of the pulse width from 60 $\mu \mathrm{s}$ to the higher values $(120,210,450 \mu \mathrm{s})$ showed a progressive reduction of the therapeutic window at every rate value $(90,130,170$ $\mathrm{Hz}$ ); these reductions were significant at $90 \mathrm{~Hz}$ increasing the pulse width value from 60 to 450 $\mu$ s and at $130 \mathrm{~Hz}$ and $170 \mathrm{~Hz}$ increasing the pulse width from 60 to $210 \mu$ s, and from 60 to $450 \mu \mathrm{s}$. For each pulse width value the largest therapeutic window was obtained using a 90 $\mathrm{Hz}$ stimulus rate, the increase of the rate to 130 and $170 \mathrm{~Hz}$ leading to a narrowing of the therapeutic window (non-significant).

\section{Discussion}

In the planning of this study we wanted to eliminate the variables unrelated to the stimulation parameters, which can interfere with the clinical evaluation. To avoid the microlesive effect of the surgical procedure (microhaemorrhage, oedema) we examined the patients at least 3 months after surgery; this time lag was considered sufficient to eliminate any clinical improvement due to the microlesive effect and to obtain a satisfactory stabilisation of the clinical picture. ${ }^{1}$ To eliminate the possible overlapping of the effect of the omolateral STN stimulation the pulse generator of the examined side was deactivated during the whole test. We solved the problem related to the persistence of a clinical effect after the interruption of the STN stimulation (after effect) by waiting, after every condition, until the baseline wrist rigidity recurred.

As a parameter of the clinical effectiveness of STN stimulation we used the subjective evaluation of contralateral wrist rigidity. This study was finalised to obtain IPWCs at different values of stimulus rate; therefore it was necessary to choose a constant clinical effect clearly detectable in every stimulation condition, strictly correlated with STN stimulation, and arising with a short latency. The disappearance of contralateral wrist rigidity in rest conditions fitted these experimental constraints well and, moreover, it strictly correlated with the effect of deep brain stimulation of the STN on all the motor symptoms of Parkinson's disease. ${ }^{12}{ }^{19}$ Tremor was not suitable for producing IPWCs because it was often fluctuating, apart from the effect of the stimulation, and because many of our patients showed a severe hypertonic-hypokinetic syndrome without tremor. The effect of STN stimulation on akinesia appears, instead, with a longer latency in respect to rigidity, making its evaluation less suitable for drawing the IPWCs.

The four different pulse width values (60, 120,210 , and $450 \mu \mathrm{s}$ ) were conditional on the range possibility offered by the pulse generator (60 to $450 \mu \mathrm{s}$ ), and they were chosen wide apart from each other to better highlight their differences in clinical effectiveness. Five stimulus rate values $(10,50,90,130,170 \mathrm{~Hz})$ were chosen to investigate the whole range of rates that can be produced by the generators, with the purpose of drawing different families of IPWCs with respect to the stimulus rate.

Previous investigations of deep brain stimulation of the STN showed, on the basis of the characteristics of the electrical stimuli, ${ }^{16} 1720$ that side effects are probably connected to the spread of the stimulus to the fibre systems near the STN (lemniscus medialis; corticospinal tract) and due to their activation. ${ }^{21} 22$

We found that the hyperbolic trends of the IPWCs for clinical effectiveness and for the onset of side effects were very similar, suggesting a common mechanism of action. This finding supports the hypothesis that the activation of inhibitory fibres afferent to the STN plays an important part in the clinical effectiveness of stimulation of the STN, as proposed by other authors for the effect of deep brain stimulation of the STN on tremor and for deep brain stimulation of the Gpi. ${ }^{21}{ }^{23}$

The trend of the IPWCs obtained at 90, 130, and $170 \mathrm{~Hz}$ was similar to the trend of the strength-duration curve of a single electrically stimulated neuronal element. ${ }^{24}$ By analysing this strength-duration curve it is possible to identify the reobase (intensity value of the stimulus under which it is not possible to obtain any neuronal effect, whatever the pulse width value used) and the chronaxie (stimulus pulse width needed to obtain a response using a stimulus intensity value equal to the double of the reobase value). ${ }^{25}$ The characteristics of the IPWCs for the clinical and side effects of deep brain stimulation of the STN, showing a striking progressive flattening of the curve at the widest pulse width values $(210 \mu$ s and $450 \mu \mathrm{s})$, are closer to the strength-duration curve typical of the stimulation of a fibre rather than of a neuronal soma; in fact, fibres present a shorter value of chronaxie (50-300 $\mu$ s) than cell bodies $(1000-3000 \mu \mathrm{s}) .^{24}{ }^{26}$ These data further underline the role of fibre activation as a possible 
mechanism of action for deep brain stimulation of the STN.

From a clinical point of view, our findings provide some information for the setting of the electrical parameters for deep brain stimulation of the STN of patients with Parkinson's disease. The increase in the pulse width led to an increase in clinical effectiveness, but considerable modifications (from $60 \mu$ s to $210 \mu$ s or $450 \mu \mathrm{s}$; from $120 \mu$ s to $450 \mu \mathrm{s}$ ) were necessary to obtain significant differences, with a consequent narrowing of the therapeutic windows.

Rate values of $10 \mathrm{~Hz}$ and $50 \mathrm{~Hz}$, even though showing in some cases a limited clinical effect, did not allow us to obtain the RCE; moreover, at these rates the increase in the intensity of the stimulus was often responsible for the onset of tremor or myoclonic jerks, with an amplitude related to the stimulus intensity.

A $90 \mathrm{~Hz}$ rate probably represents the threshold for the clinical effectiveness: the RCE was in fact obtained in some patients only with the narrower pulse width values. In some cases increasing the stimulus intensity made it possible to see the onset of side effects similar to those typical of lower rate values $(10 \mathrm{~Hz}$ and 50 $\mathrm{Hz}$ ), as tremor or myoclonic jerks.

The stimulation at 130 or $170 \mathrm{~Hz}$ was effective in all the patients for every pulse width value. The clinical effectiveness was similar at 130 and $170 \mathrm{~Hz}$ was comparable with the therapeutic windows. The side effects found when increasing the stimulus intensity at 130 and $170 \mathrm{~Hz}$ were paraesthesias, muscle contractions, and dyskinesias, and they were different from those at 10 and $50 \mathrm{~Hz}$, probably reflecting a different mechanism of action of the electrical stimulation at different rate values.

It seems therefore for deep brain stimulation of the STN that a stimulus rate higher than 90 $\mathrm{Hz}$ gives the best clinical effects, whereas, above this value, there are no significant advantages in increasing the stimulus rate up to $170 \mathrm{~Hz}$. Moreover, the slight improvement in clinical effectiveness obtainable using pulse width values larger than $60 \mu \mathrm{s}$ is accompanied by a significant narrowing of the therapeutic window; therefore, pulse width values larger than $60 \mu$ s are probably useful only in selected patients, when the clinical effect is not satisfactory with narrower values. Finally, the finding of a significant improvement of tremor and akinesia in all the different experimental conditions when the RCE on rigidity was reached could suggest a similar effectiveness of deep brain stimulation of the STN on cardinal
Parkinson's disease symptoms with similar therapeutic windows. ${ }^{1-3} 1419$

1 Limousin P, Krack P, Pollak P, et al. Electrical stimulation of the subthalamic nucleus in advanced Parkinson's disease. $N$ Engl f Med 1998;339:1105-11.

2 Lopiano L, Rizzone M, Bergamasco B, et al. Deep brain stimulation of the subthalamic nucleus: clinical effectiveness and safety. Neurology 2001;56:552-4.

3 Moro E, Scerrati M, Romito LM, et al. Chronic subthalamic nucleus stimulation reduces medication requirements in Parkinson's disease. Neurology 1999;53:85-90.

4 Kumar R, Lozano AM, Sime E, et al. Comparative effects of unilateral and bilateral subthalamic nucleus deep brain stimulation. Neurology 1999;53:561-6.

5 Parent A. Extrinsic connections of the basal ganglia. Trends Neurosci 1990;13:254-8.

6 Alexander GE, Crutcher MD. Functional architecture of basal ganglia circuits: neural substrates of parallel processbasal ganglia circuits: neural substrates

7 Levy R, Hazrati L-N, Herrero MT, et al. Re-evaluation of the functional anatomy of the basal ganglia in normal and parkinsonian states [review]. Neuroscience 1997;76:335-43.

8 Bergman H, Wichmann T, DeLong MR. Reversal of experimental parkinsonism by lesions of the subthalamic nucleus. Science 1990;249:1436-8.

9 Benazzouz A, Gross C, Feger J, et al. Reversal of rigidity and improvement in motor performance by subthalamic high-frequency stimulation in MPTP-treated monkeys. Eur 7 Neurosci 1993;5:382-9.

10 Benabid AL, Benazzouz A, Hoffmann D, et al. Long-term electrical inhibition of deep brain targets in movement disorders. Mov Disord 1998;13:119-25.

11 Benabid AL, Pollak P, Gao D, et al. Chronic electrical stimulation of the ventralis intermedius nucleus of the thalamus as a treatment of movement disorders. I Neurosurg 1996;84:203-14.

12 Hutchinson WD, Allan RJ, Opitz H, et al. Neurophysiologial identification of the subthalamic nucleus in surgery for Parkinson's disease. Ann Neurol 1998;44:622-8

13 Limousin P, Pollak P, Benazzouz A, et al. Effect of parkinsonian signs and symptoms of bilateral subthalamic nucleus stimulation. Lancet 1995;345:91-5.

14 Pollak P, Benabid AL, Krack P, et al. Deep brain stimulation. n: Parkinson's disease and movement disorders. Baltimore: Williams and Wilkins, 1998;48:1085-101.

15 O'Mara S, Rowe MJ, Tarvin RP. Neural mechanisms in vibrotactile adaptation. $\mathcal{F}$ Neurophysiol 1988;59:607-22

16 Nowak LG, Bullier J. Axons, but not cell bodies, are activated by electrical stimulation in cortical gray matter. I. Evidence from selective inactivation of cell bodies and axon initial segments. Exp Brain Res 1998;118:489-500.

17 Nowak LG, Bullier J. Axons, but not cell bodies, are activated by electrical stimulation in cortical gray matter. I. Evidence from chronaxie measurements. Exp Brain Res 1998b;118:477-88.

18 Limousin P, Pollak P, Hoffmann D, et al. Abnormal involuntary movements induced by subthalamic nucleus stimulation in parkinsonian patients. Mov Disord 1996;11:231-5.

19 Limousin P, Pollak P, Benazzouz A, et al. Bilateral subthalamic nucleus stimulation for severe Parkinson's disease. Mov Disord 1995;10:672-4.

20 Ranck JB. Extracellular stimulation. In: Patterson MM, Kesner RP, eds. Electrical stimulation research techniques. New York: Academic Press, 1981:2-36.

21 Ashby P, Kim YJ, Kumar R, et al. Neurophysiological effects of stimulation through electrodes in the human subthalamic nucleus. Brain 1999;122:1919-31.

22 Ashby P, Strafella A, Dostrovsky JO, et al. Immediate motor effects of stimulation through electrodes implanted in the human globus pallidus. Stereotact Funct Neurosurg 1998;70: $1-18$.

23 Ashby P, Wu YR, Levy R, et al. Does stimulation of the Gpi control dyskinesia by activating inhibitory axons? Neurolcontrol dyskinesia by activatin

24 Tehy 2000;54(suppl 3):A284 behavioral responses. $\mathcal{F}$ Neurosci Methods 1996;65:1-17.

25 Jayakar P. Physiological principles of electrical stimulation. In: O Devinsky, ed. Electrical and magnetic stimulation of the brain and spinal cord. New York: Raven Press, 17:1993

26 Ranck JB. Which elements are excited in electrical stimulation of mammalian central nervous system: a review. Brain Res 1975;98:417-40. 